\title{
Combining Ability and Gene Action in Cucumber (Cucumis Sativus L.)
}

\author{
S. Ahammed, \\ Senior Assistant Director, Bangladesh Agricultural Development Corporation
}

(BADC), Tebunia, Pabna.

M. M. Hossain,

Professor, Department of Horticulture, Bangabandhu Sheikh Mujibur Rahman Agricultural University (BSMRAU), Salna, Gazipur

\section{Zakaria,}

Professor, Department of Horticulture, Bangabandhu Sheikh Mujibur Rahman Agricultural University (BSMRAU), Salna, Gazipur.

\section{B. Ahmed (Corresponding author)}

Senior Scientific Officer, HRC, Bangladesh Agricultural Research Institute (BARI), Gazipur.

$$
\text { M.A.K. Mian }
$$

Professor, Department of Genetics and Plant Breeding, Bangabandhu Sheikh Mujibur Rahman Agricultural University (BSMRAU), Salna, Gazipur.

Received: May 18, 2018

doi:10.5296/jas.v6i2.13329
Accepted: June 5, 2018

URL: https://doi.org/10.5296/jas.v6i2.13329 


\section{Abstract}

Combining ability and genetic components of eleven inbred line of cucumber were estimated following line $\mathrm{x}$ tester mating design for qualitative and quantitative characters. Three inbred lines were used as tester. Variance within the treatments, parents, parent vs crosses, crosses, testers and line $\mathrm{x}$ tester interaction were highly significant for all the characters. Considering the gca effects the lines CS08, CS16, CS040, CS07 and CS51 were best for their earliness and other horticulture traits. The hybrids CS07×CS08, CS16×CS44, CS51×CS44, CS40 $\times$ CS08, CS17 $\times$ CS39 were superior in terms of yield per plant and its component characters. The magnitude of $\sigma^{2} \mathrm{SCA}$ was high in all characters compared to $\sigma^{2} \mathrm{GCA}$ and dominance variance $\left(\sigma^{2} \mathrm{D}\right)$ was higher than the additive genetic variance $\left(\sigma^{2} \mathrm{~A}\right)$ indicating that the predominance role of non-additive gene action. The results indicated the importance of heterosis breeding for effective utilization of non-additive genetic variance in cucumber.

Keywords: Cucumber, combining ability, gca, sca

\section{Introduction}

Cucumber (Cucumis sativus L.) belongs to the family cucurbitaceae and its centre of origin is India (Renner et al., 2007). It is an important and one of the most popular fruit vegetable in Asia. Cucumber is also an ideal vegetable for its edible tender fruits, preferred as salad ingredient, pickles, desert fruit and as a cooked vegetable. The fruit has about $95 \%$ water content (Anonymous, 2012) which makes it diuretic, possessing a deep cleansing action due to the presence of some natural chemical constituents such as glycolic, lactic and salicylic acids (Uzodike and Onuoha, 2009). Cucumber is grown widely in different parts of the world. It is year round out door vegetable in the tropics (Eifediyi and Remison, 2010). Since there exist a considerable variability of this crop, it is possible to develop high yielding varieties through breeding approaches like selection or hybridization.

Combining ability is one of the important and powerful tools to identifying the best combiner that may be used in crosses to exploit heterosis. It helps to know the genetic architecture of various characters that enable the breeder to design effective breeding plan for future improvement of the existing materials. The combining ability analysis gives useful information regarding the selection of parents in terms of the performance of their hybrids. Information on the relative importance of general combining ability (GCA) and specific combining ability (SCA) is of value in breeding programs for species which are amenable to the development of $F_{1}$ hybrid cultivars such basic information on combining ability in cucumber would aid the breeder in developing improved hybrid cultivars (Tasdighi and Baker, 1981). The line $\mathrm{x}$ tester technique was developed by Kempthorne in 1957. It is a good approach for screening the germplasm on the basis of GCA and SCA variances and effects. Line $\times$ tester analysis involving parents give the additional information about the presence or absence of epistasis, average degree of dominance, as well as distribution of dominant and recessive genes in the parents. Application of line $\times$ tester technique in a cross-pollinated crop like cucumber for this purpose may be appropriate. The estimates of gene effects and genetic variance help in understanding the genetic potential of the breeding material. Fruit yield being a polygenic character, many genes are involved in its inheritance. Because of the 
small effect of individual genes, it is apparently difficult to study their individual effects. The present investigation was undertaken to generate information for identification of good general and specific combiners of cucumber genotypes for the improvement of yield and its attributes.

\section{Materials and Methods}

The experiment was conducted at the research farm of Horticulture Department, Bangabandhu Sheikh Mujibur Rahman Agricultural University, Gazipur during March, 2013 to June, 2013. The study consisted of eight inbred lines as female parent and three testers as male parent with diverse genetic base. The parents were selected based on their performance on different horticultural traits, heritability and genetic diversity as evaluated in earlier experiment. The experiment was laid out in randomized complete block design (RCBD) with three replications. Twenty four cross combinations were produced through line $\mathrm{x}$ tester mating design in previous year and together with their eleven parents (CS07, CS08, CS16, CS17, CS18, CS39, CS40, CS44, CS50, CS51 and CS52). The characteristics of selected 11 parents are presented in Table 1. The genotypes were randomly allotted in each block. The unit plot size was $1.5 \times 3.75 \mathrm{~m}$ accommodating 10 plants in each plot having row and plant spacing of $1 \mathrm{~m} \times 0.75 \mathrm{~m}$, respectively. Adjacent plots were separated by $50 \mathrm{~cm}$ and while blocks were separated by $1.0 \mathrm{~m}$.

Chemical fertilizers 69-35-75-5.5-4.3-3.25 kg/ha of N-P-K-S-Zn-B and 10 tons/ha cow-dung were applied (Fertilizer Recommendation Guide, 2012). Half of cow-dung and MoP and the entire amount of TSP, Gypsum, Zinc oxide and Boric acid were applied in the field during final land preparation. The remaining cow-dung, MoP and one third of urea were applied in the pit one week prior to transplanting. Twenty days old seedlings were transplanted. Top dressings were done in the two equal installments at 20 and 40 days after transplanting. Plants were given support by bamboo sticks for climbing on trellis. Trellis were made by bamboo sticks to support the growing plants and allowed them to creep. The seedlings were watered immediately after transplanting. Necessary intercultural operations were done to ensure normal growth and development of the plants. Adult red pumpkin beetles were controlled by applying ripcord @ of $10 \mathrm{ml}$ per 10 liters of water. At fruiting stage to control the attack of fruit fly, pheromone trap were used at a density of $5 \mathrm{~m}^{2} /$ trap. Five random competitive plants were selected and tagged from each plot. Observations were recorded on these plants on days to $1^{\text {st }}$ male flower, days to $1^{\text {st }}$ female flower, node at $1^{\text {st }}$ male flower appearance, node at $1^{\text {st }}$ female flower appearance, days to $50 \%$ male flower, days to $50 \%$ female flower, days to $1^{\text {st }}$ fruit harvest, vine length, number of branches per plant, fruit length, fruit width, flesh thickness, single fruit weight, number of fruits per plant, yield per plant and 1000 seed weight. 


\subsection{Statistical Analysis}

All the quantitative data taken were subjected to ANOVA and differences tested by F-test. The combining ability analysis were done according to Kempthorne (1957) based on the performance of the parents and their relative contribution to the $\mathrm{F}_{1} \mathrm{~s}$ as determined by the general combining ability effects and specific combining ability effects. For analysis of data MS-EXCEL and R i386 3.0.1 statistical software were used.

Table 1. Characteristics of selected 11 inbred lines of cucumber

\begin{tabular}{|c|c|c|c|c|c|c|c|c|c|c|c|}
\hline Characters & CS07 & CS08 & CS16 & CS17 & CS18 & CS39 & CS40 & CS44 & CS50 & CS51 & CS52 \\
\hline $\begin{array}{l}\text { Days to } 1^{\text {st }} \\
\text { male flower }\end{array}$ & 43.4 & 42.6 & 42.8 & 43.1 & 42.9 & 43.7 & 43.5 & 42.1 & 41.5 & 48.5 & 43.8 \\
\hline $\begin{array}{l}\text { Node at } 1^{\text {st }} \\
\text { male flower } \\
\text { appearances }\end{array}$ & 3.9 & 4.3 & 4.3 & 5.1 & 6.1 & 5.1 & 4.6 & 3.9 & 4.7 & 6.4 & 3.9 \\
\hline $\begin{array}{l}\text { Days to } 1^{\text {st }} \\
\text { female flower }\end{array}$ & 46.5 & 44.7 & 45.3 & 44.9 & 44.9 & 46.7 & 45.8 & 43.7 & 45.7 & 55.6 & 46.3 \\
\hline $\begin{array}{l}\text { Node at } 1^{\text {st }} \\
\text { female flower } \\
\text { appearances }\end{array}$ & 6.2 & 6.5 & 6.4 & 6.1 & 7.9 & 7.4 & 6.9 & 4.8 & 7.1 & 8.9 & 6.7 \\
\hline $\begin{array}{l}\text { Days to } 50 \% \\
\text { plants male } \\
\text { flower }\end{array}$ & 45.0 & 43.7 & 44.3 & 44.0 & 44.7 & 44.7 & 45.0 & 43.7 & 45.0 & 51.0 & 46.0 \\
\hline $\begin{array}{l}\text { Vine length } \\
(\mathrm{cm})\end{array}$ & 339.1 & 343.6 & 351.1 & 332.0 & 340.1 & 175.8 & 304.4 & 152.8 & 357.1 & 312.5 & 273.5 \\
\hline $\begin{array}{l}\text { No. of } \\
\text { branches/plant }\end{array}$ & 9.3 & 8.2 & 6.7 & 7.9 & 8.8 & 9.3 & 9.2 & 8.9 & 7.9 & 7.0 & 5.1 \\
\hline $\begin{array}{l}\text { No. of } \\
\text { fruits/plant }\end{array}$ & 8.0 & 7.0 & 6.7 & 7.2 & 5.9 & 8.9 & 8.1 & 9.8 & 7.3 & 5.3 & 5.0 \\
\hline $\begin{array}{l}\text { Days to } 1^{\text {st }} \\
\text { fruit harvest }\end{array}$ & 59.6 & 57.7 & 58.5 & 57.6 & 57.9 & 59.1 & 58.3 & 57.0 & 56.7 & 68.7 & 57.9 \\
\hline $\begin{array}{l}\text { Fruit length } \\
(\mathrm{cm})\end{array}$ & 16.4 & 18.5 & 18.9 & 20.2 & 20.3 & 11.7 & 20.0 & 13.3 & 20.3 & 22.9 & 12.6 \\
\hline $\begin{array}{l}\text { Yield per } \\
\text { plant (kg) }\end{array}$ & 2.7 & 2.1 & 2.9 & 2.7 & 2.0 & 1.5 & 1.8 & 1.5 & 2.1 & 2.0 & 0.9 \\
\hline $\begin{array}{l}1000 \text { seed } \\
\text { weight }(\mathrm{gm})\end{array}$ & 26.8 & 26.3 & 27.2 & 28.9 & 30.0 & 23.9 & 28.8 & 26.6 & 23.6 & 28.2 & 23.5 \\
\hline
\end{tabular}




\section{Results and Discussion}

The analysis of variance for combining ability based on mean square of different characters in 11 cucumber inbred lines was presented in Table 2. Variances within the treatments, parents, parent vs crosses, crosses, testers and line $\times$ tester interaction were highly significant for all the characters. Variance due to line was significant for days to $1^{\text {st }}$ male flower and days to $50 \%$ male flower. Variance due to tester was significant for most of the characters except node at $1^{\text {st }}$ male flower appearances, node at $1^{\text {st }}$ female flower appearances, flesh thickness and yield per plant which indicates that both additive and no-additive gene actions played significant role for expression of these characters.

Table 2. Analysis of variance based on mean squares and estimates of genetic component in line $\times$ tester study of cucumber

\begin{tabular}{|c|c|c|c|c|c|c|c|c|c|}
\hline $\begin{array}{l}\text { Source of } \\
\text { variation }\end{array}$ & $\mathrm{df}$ & $\begin{array}{l}\text { Days to } \\
1^{\text {st }} \text { male } \\
\text { flower }\end{array}$ & $\begin{array}{l}\text { Node at } 1^{\text {st }} \\
\text { male flower } \\
\text { appearances }\end{array}$ & $\begin{array}{l}\text { Days to } \\
1^{\text {st }} \\
\text { female } \\
\text { flower }\end{array}$ & $\begin{array}{l}\text { Node at } 1^{\text {st }} \\
\text { female } \\
\text { flower } \\
\text { appearances }\end{array}$ & $\begin{array}{l}\text { Days to } \\
50 \% \\
\text { plant } \\
\text { male } \\
\text { flower }\end{array}$ & $\begin{array}{l}\text { Days to } \\
50 \% \\
\text { plant } \\
\text { female } \\
\text { flower }\end{array}$ & $\begin{array}{l}\text { Vine length } \\
(\mathrm{cm})\end{array}$ & $\begin{array}{l}\text { No. of } \\
\text { branches } \\
\text { per plant }\end{array}$ \\
\hline Replication & 2 & 1.23 & 0.35 & 0.15 & 0.63 & 1.67 & 3.09 & 1118.99 & $0.76^{*}$ \\
\hline Treatments & 34 & $41.99 * *$ & $1.74 * *$ & $58.17 * *$ & $8.37 * *$ & $47.53 * *$ & $72.92 * *$ & $12940.99 * *$ & $12.45^{* *}$ \\
\hline Parents & 10 & $10.05^{* *}$ & $2.21 * *$ & $30.41 * *$ & 3.41 & $12.56 * *$ & $43.61 * *$ & $14963.38 * *$ & $5.13^{* *}$ \\
\hline $\begin{array}{l}\text { Parents vs. } \\
\text { Crosses }\end{array}$ & 1 & $165.95 * *$ & $4.59 * *$ & $25.65 * *$ & $49.47 * *$ & $83.24 * *$ & 0.002 & 6478.74 & $29.00 * *$ \\
\hline Crosses & 23 & $50.50 * *$ & $1.41 * *$ & $71.65^{* *}$ & $8.74 * *$ & $61.19 * *$ & $88.83 * *$ & $12342.66 * *$ & $14.92 * *$ \\
\hline Line & 7 & $63.56^{*}$ & 0.76 & 65.54 & 7.99 & $77.49 *$ & 81.46 & 6406.53 & 5.97 \\
\hline Tester & 2 & $187.30 * *$ & 2.62 & $309.93 * *$ & 11.90 & $207.10 * *$ & $353.84 * *$ & $79498.45^{* *}$ & $76.24 * *$ \\
\hline $\begin{array}{l}\text { Line } \\
\text { tester }\end{array}$ & 14 & $24.42 * *$ & $1.57 * *$ & $40.67 * *$ & $8.66 * *$ & $32.19 * *$ & $54.65 * *$ & $5717.04 * *$ & $10.63 * *$ \\
\hline Error & 68 & 1.15 & 0.13 & 1.88 & 2.05 & 1.75 & 1.22 & 165.53 & 0.25 \\
\hline
\end{tabular}

Table 2. Cont`d

\begin{tabular}{|c|c|c|c|c|c|c|c|c|c|}
\hline $\begin{array}{l}\text { Source of } \\
\text { variation }\end{array}$ & $\mathrm{df}$ & $\begin{array}{l}\text { No. of } \\
\text { fruits } \\
\text { per } \\
\text { plant }\end{array}$ & $\begin{array}{l}\text { Days to } \\
1^{\text {st }} \text { fruit } \\
\text { harvest }\end{array}$ & $\begin{array}{l}\text { Fruit } \\
\text { length } \\
(\mathrm{cm})\end{array}$ & $\begin{array}{l}\text { Fruit } \\
\text { width } \\
(\mathrm{cm})\end{array}$ & $\begin{array}{l}\text { Flesh } \\
\text { thickness } \\
(\mathrm{cm})\end{array}$ & $\begin{array}{l}\text { Single fruit } \\
\text { weight }(\mathrm{g})\end{array}$ & $\begin{array}{l}\text { Yield } \\
\text { per } \\
\text { plant } \\
(\mathrm{kg})\end{array}$ & $\begin{array}{l}1000 \\
\text { seed } \\
\text { weight } \\
(\mathrm{g})\end{array}$ \\
\hline Replication & 2 & 0.19 & 2.37 & 0.67 & 0.02 & 0.03 & 13.69 & 0.08 & 1.72 \\
\hline Treatments & 34 & $7.80 * *$ & $72.03 * *$ & $34.20 * *$ & $1.37 * *$ & $0.33 * *$ & $15723.23^{* *}$ & $0.88 * *$ & $25.89 * *$ \\
\hline Parents & 10 & $6.54 * *$ & $33.04 * *$ & $40.90 * *$ & $1.57 * *$ & $0.36 * *$ & $26226.09 * *$ & $0.33 * *$ & $15.26 * *$ \\
\hline $\begin{array}{l}\text { Parents vs. } \\
\text { crosses }\end{array}$ & 1 & $78.84 * *$ & $104.50 * *$ & $216.00 * *$ & $2.42 * *$ & $0.54 * *$ & $69176.19^{* *}$ & $4.42 * *$ & 0.87 \\
\hline Crosses & 23 & $5.26 * *$ & $87.58 * *$ & $23.39 * *$ & $1.24 * *$ & $0.30 * *$ & $8832.72 * *$ & $0.97 * *$ & $31.60 * *$ \\
\hline Line & 7 & 2.70 & 78.73 & 6.29 & 0.38 & 0.13 & 5144.69 & 0.57 & 39.92 \\
\hline Tester & 2 & $29.75^{* *}$ & $348.37 * *$ & $138.31 * *$ & $5.22 *$ & 0.71 & $39322.18^{* *}$ & 5.63 & $64.78^{*}$ \\
\hline $\begin{array}{l}\text { Line } \quad \times \\
\text { tester }\end{array}$ & 14 & $3.05 * *$ & $54.75^{* *}$ & $15.52 * *$ & $1.10 * *$ & $0.33^{* *}$ & $6321.10 * *$ & $0.50 * *$ & $22.70^{* *}$ \\
\hline Error & 68 & 0.17 & 1.01 & 0.53 & 0.03 & 0.01 & 76.99 & 0.06 & 2.53 \\
\hline
\end{tabular}




\section{General Combining Ability Effects}

The GCA variances of each parent play significant role for the choice of parents. A parent with higher positive significant gca effects is considered as a good general combiner. The magnitude and direction of the significant effects for the eleven parents provide meaningful comparisons and would give indications to the future breeding program.

Considering gca effects testers CS08 and CS44 were the best for days to $1^{\text {st }}$ male and female flower, node at $1^{\text {st }}$ male and female flower appearances, days to $50 \%$ plants male and female flower, vine length, number of branches per plant, number of fruits per plant, days to $1^{\text {st }}$ fruit harvest, fruit length, single fruit weight and yield per plant (Table 3.1). The parental lines CS16 was the best for days to $1^{\text {st }}$ male and female flower, days to $50 \%$ male and female flower, number of fruits per plant, days to $1^{\text {st }}$ fruit harvest, fruit length, fruit width, flesh thickness, yield per plant and thousand seed weight, CS17 was the best for days to $1^{\text {st }}$ male and female flower, days to $50 \%$ male and female flower, vine length, number of branches per plant, days to $1^{\text {st }}$ fruit harvest, fruit length, single fruit weight and thousand seed weight, CS40 was the best for days to $1^{\text {st }}$ male and female flower, node at $1^{\text {st }}$ male and female flower appearances, days to $50 \%$ male and female flower, days to $1^{\text {st }}$ fruit harvest, single fruit weight, CS51 was the best for vine length, fruit length, fruit width, flesh thickness, single fruit weight, yield per plant and thousand seed weight, CS07 was best for number of fruits per plant, single fruit weight and yield per plant; CS50 was the flesh thickness and yield per plant and CS18 was the best for vine length and single fruit weight (Table 3.1, 3.2 and 3.3). These indicated that, CS44, CS08, CS16, CS51, CS40 and CS07 may contribute significant to the performance of their progenies. Krishnaprasad and Singh (1992) reported the two parents were good general combiners for yield and fruit number. Ananthan and Pappiah (1997) reported that general combining ability were significant for days to first male flower, days to first female flower, fruit number per plant, fruit length, fruit girth, and single fruit weight. Similar results were also reported by Verma et al. (2000) in days to $50 \%$ male and female flowers, fruit length, weight and girth, number of fruits per plant and fruit yield per plant. 
Table 3.1. gca effects of flowering behavior of cucumber

\begin{tabular}{|c|c|c|c|c|c|c|}
\hline Parents & $\begin{array}{l}\text { Days to } 1^{\text {st }} \\
\text { male flower }\end{array}$ & $\begin{array}{l}\text { Days to } 1^{\text {st }} \\
\text { female flower }\end{array}$ & $\begin{array}{l}\text { Node at } 1^{\text {st }} \text { male } \\
\text { flower appearance }\end{array}$ & $\begin{array}{l}\text { Node at } 1^{\text {st }} \text { female } \\
\text { flower appearance }\end{array}$ & $\begin{array}{ll}\begin{array}{l}\text { Days to } \\
\text { plant }\end{array} & \text { male } \\
\text { flower } & \\
\end{array}$ & $\begin{array}{l}\text { Days to } 50 \% \\
\text { plant } \\
\text { flower }\end{array}$ \\
\hline \multicolumn{7}{|l|}{ Lines } \\
\hline CS07 & 0.32 & 0.31 & -0.04 & 0.47 & 0.18 & $0.78 *$ \\
\hline CS16 & $-1.54 * *$ & $-1.64 * *$ & -0.01 & -0.04 & $-1.71 * *$ & $-1.67 * *$ \\
\hline CS17 & $-1.63^{* *}$ & $-1.67 * *$ & $0.21 *$ & -0.59 & $-1.71 * *$ & $-2.33 * *$ \\
\hline CS18 & $1.61 * *$ & $1.73 * *$ & 0.12 & -0.10 & $1.74 * *$ & $1.56^{* *}$ \\
\hline CS40 & $-4.94 * *$ & $-5.22 * *$ & $-0.68 * *$ & $-1.75 * *$ & $-5.37 * *$ & $-5.67 * *$ \\
\hline CS50 & $2.46^{* *}$ & $2.33 * *$ & 0.14 & $1.52 * *$ & $2.40 * *$ & $2.67 * *$ \\
\hline CS51 & $3.32 * *$ & $2.80 * *$ & $0.21 *$ & -0.04 & $4.07 * *$ & $3.33 * *$ \\
\hline CS52 & 0.41 & $1.36 * *$ & 0.05 & 0.52 & 0.40 & $1.33 * *$ \\
\hline SE gca & 0.35 & 0.45 & 0.12 & 0.48 & 0.44 & 0.37 \\
\hline SE (gi-gj) & 0.51 & 0.65 & 0.17 & 0.67 & 0.62 & 0.52 \\
\hline \multicolumn{7}{|l|}{ Testers } \\
\hline CS08 & $-0.91 * *$ & $-1.13 * *$ & 0.04 & $0.51 *$ & $-1.06^{* * *}$ & $-1.24 * *$ \\
\hline CS39 & $3.14 * *$ & $4.02 * *$ & $0.31 * *$ & 0.29 & $3.32 * *$ & $4.31 * *$ \\
\hline CS44 & $-2.22 * *$ & $-2.89 * *$ & $-0.35 * *$ & $-0.80 * *$ & $-2.26 * *$ & $-3.07 * *$ \\
\hline SE gca & 0.22 & 0.28 & 0.07 & 0.29 & 0.27 & 0.22 \\
\hline $\begin{array}{l}\text { SE } \\
\text { (gi-gj) }\end{array}$ & 0.31 & 0.39 & 0.11 & 0.41 & 0.38 & 0.32 \\
\hline
\end{tabular}

Table 3.2. gca effects for days to 1st fruit harvest, number of branches per plant, vine length, fruit length and fruit width of cucumber

Vine length Fruit length Fruit width

Parents Days to $1^{\text {st }}$ fruit harvest No. of branches per plant

\begin{tabular}{lccccc}
\hline Lines & \multicolumn{1}{c}{} & \\
\hline CS07 & -0.03 & 0.29 & -2.39 & $-0.48^{*}$ & $-0.24^{* *}$ \\
CS16 & $-1.82^{* *}$ & 0.28 & 2.67 & -0.32 & $0.31^{* *}$ \\
CS17 & $-1.77^{* *}$ & $0.75^{* *}$ & $28.81^{* *}$ & $0.75^{* *}$ & $-0.12^{*}$ \\
CS18 & $1.29^{* *}$ & $0.97^{* *}$ & $22.54^{* *}$ & -0.41 & $-0.14^{*}$ \\
CS40 & $-5.63^{* *}$ & $-0.59^{* *}$ & $-28.19^{* *}$ & $0.69^{* *}$ & 0.05 \\
CS50 & $2.78^{* *}$ & $-1.50^{* *}$ & 0.24 & -0.28 & 0.01 \\
CS51 & $3.01^{* *}$ & $-0.54^{* *}$ & $23.92^{* *}$ & $1.31^{* *}$ & $0.28^{* *}$ \\
CS52 & $2.16^{* *}$ & $0.33^{*}$ & $-47.59^{* *}$ & $-1.25^{* *}$ & $-0.14^{*}$ \\
\hline SE gca & 0.33 & 0.17 & 4.29 & 0.24 & 0.06 \\
SE (gi-gj) & 0.47 & 0.23 & 6.06 & 0.34 & 0.08 \\
\hline Testers & & & & & \\
\hline CS08 & $-1.57^{* *}$ & $0.23^{*}$ & $32.62^{* *}$ & $2.60^{* *}$ & -0.002 \\
CS39 & $4.34 * *$ & $-1.89^{* *}$ & $-66.45^{* *}$ & $-2.13^{* *}$ & $0.47^{* *}$ \\
CS44 & $-2.77^{* *}$ & $1.65^{* *}$ & $33.84^{* *}$ & $-0.47^{* *}$ & $-0.46^{* *}$ \\
\hline SE gca & 0.20 & 0.10 & 2.63 & 0.15 & 0.03 \\
SE (gi-gj) & 0.29 & 0.14 & 3.71 & 0.21 & 0.05 \\
\hline
\end{tabular}

$*$ and $* *$ significant at $5 \%$ and $1 \%$ level, respectively 
Table 3.3. gca effects for yield, yield contributing traits and thousand seed weight of cucumber

\begin{tabular}{|c|c|c|c|c|c|}
\hline Parents & No. of fruits per plant & Fruit flesh thickness & Single fruit weight & Yield per plant & Thousand seed weight \\
\hline \multicolumn{6}{|l|}{ Lines } \\
\hline CS07 & $0.67 * *$ & $-0.07 * *$ & $10.87 * *$ & $0.35 * *$ & -0.87 \\
\hline CS16 & $0.80 * *$ & $0.08 * *$ & $-5.62 *$ & $0.18 * *$ & $2.95^{* *}$ \\
\hline CS17 & $-0.47 * *$ & $-0.08 * *$ & $13.63 * *$ & $-0.22 * *$ & $1.99 * *$ \\
\hline CS18 & -0.07 & -0.02 & $15.52 * *$ & $-0.15^{*}$ & $-2.75 * *$ \\
\hline CS40 & 0.20 & $-0.12 * *$ & $7.34 *$ & 0.05 & $-1.42 * *$ \\
\hline CS50 & $-0.33^{*}$ & $0.15^{* *}$ & 1.36 & $0.013^{*}$ & $-1.07 *$ \\
\hline CS51 & 0.00 & $0.17 * *$ & $13.38 * *$ & $0.21 * *$ & $2.33 * *$ \\
\hline CS52 & $-0.80 * *$ & $-0.13 * *$ & $-56.48 * *$ & $-0.42 * *$ & $-1.17 *$ \\
\hline SE gca & 0.13 & 0.03 & 2.92 & 0.07 & 0.53 \\
\hline SE (gi-gj) & 0.19 & 0.05 & 4.14 & 0.11 & 0.75 \\
\hline \multicolumn{6}{|l|}{ Testers } \\
\hline CS08 & $0.15^{*}$ & $-0.10 * *$ & $18.16^{* *}$ & $0.36 * *$ & $0.61 *$ \\
\hline CS39 & $-1.8 * *$ & $0.20 * *$ & $-46.38 * *$ & $-0.55^{* *}$ & $-1.86 * *$ \\
\hline CS44 & $1.03 * *$ & $-0.10 * *$ & $28.21 * *$ & $0.19 * *$ & $1.25^{* *}$ \\
\hline SE gca & 0.08 & 0.02 & 1.79 & 0.05 & 0.32 \\
\hline SE (gi-gj) & 0.11 & 0.03 & 2.53 & 0.07 & 0.46 \\
\hline
\end{tabular}

$*$ and $* *$ significant at $5 \%$ and $1 \%$ level, respectively

\section{Specific combining ability (sca) effects}

The sca effects signify the role of non-additive gene action in the expression of the characters. It indicates the high specific combining ability leading to highest performance of some specific cross combinations. That is why it is related to a particular cross. High sca effects may arise not only in crosses involving high $\times$ high combination but also in those involving low $\times$ high and low $\times$ low combination. Thus in practice, some of the low combiners should also be accommodated in hybridization program. Estimates on sca effects of the crosses in $\mathrm{F}_{1}$ generation showed that there were a good number of crosses having significant positive or negative sca effect for different important characters of cucumber. None of the hybrids indicated significant positive sca effects for all the characters. Sharma et al. (2001) and Ram et al. (1999) reported similar results in the case of cucumber and bitter gourd, respectively. 


\section{Al Macrothink}

Journal of Agricultural Studies

ISSN 2166-0379

2018, Vol. 6, No. 2

Table 4.1. Estimates of sca effects of crosses for flowering behavior of cucumber

\begin{tabular}{|c|c|c|c|c|c|c|}
\hline Crosses & $\begin{array}{l}\text { Days to } 1^{\text {st }} \text { male } \\
\text { flower }\end{array}$ & $\begin{array}{l}\text { Days to } 1^{\text {st }} \text { female } \\
\text { flower }\end{array}$ & $\begin{array}{l}\text { Node at } 1^{\text {st }} \text { male } \\
\text { flower appearance }\end{array}$ & $\begin{array}{l}\text { Node at } 1^{\text {st }} \text { female } \\
\text { flower appearance }\end{array}$ & $\begin{array}{l}\text { Days to } 50 \% \text { plants } \\
\text { male lower }\end{array}$ & $\begin{array}{l}\text { Days to } 50 \% \text { plants } \\
\text { female flower }\end{array}$ \\
\hline $\mathrm{CS} 07 \times \mathrm{CS} 08$ & $-3.33 * *$ & $-3.63 * *$ & $-1.48 * *$ & $-3.09 * *$ & $-4.06 * *$ & $-4.65 * *$ \\
\hline CS07×CS39 & $4.75 * *$ & $6.29 * *$ & $0.78 * *$ & 0.67 & $5.24 * *$ & $6.14 * *$ \\
\hline $\mathrm{CS} 07 \times \mathrm{CS} 44$ & $-1.42 *$ & $-2.66^{* *}$ & $0.70 * *$ & $2.43 * *$ & -1.18 & $-1.49^{*}$ \\
\hline CS16×CS08 & 0.80 & -0.81 & $1.03 * *$ & 0.29 & 0.50 & -1.21 \\
\hline CS16×CS39 & 0.62 & $2.31 * *$ & 0.09 & 0.84 & 1.13 & $2.92 * *$ \\
\hline CS16×CS44 & $-1.42 *$ & -1.51 & $-1.12 * *$ & -1.13 & $-1.63^{*}$ & $-1.71 * *$ \\
\hline $\mathrm{CS} 17 \times \mathrm{CS} 08$ & 0.29 & 0.08 & 0.08 & -0.23 & 0.83 & 0.46 \\
\hline CS17×CS39 & $-2.83 * *$ & $-3.86^{* *}$ & -0.40 & -0.33 & $-3.54 * *$ & $-4.75^{* *}$ \\
\hline $\mathrm{CS} 17 \times \mathrm{CS} 44$ & $2.53 * *$ & $3.78 * *$ & 0.33 & 0.56 & $2.71 * *$ & $4.29 * *$ \\
\hline $\mathrm{CS} 18 \times \mathrm{CS} 08$ & 0.65 & 0.08 & -0.17 & -0.05 & 0.39 & -0.10 \\
\hline CS18×CS39 & 1.00 & 1.53 & 0.02 & 0.18 & 1.01 & $2.03 * *$ \\
\hline $\mathrm{CS} 18 \times \mathrm{CS} 44$ & $-1.64 * *$ & $-1.62 *$ & 0.15 & -0.13 & -1.40 & $-1.93 * *$ \\
\hline $\mathrm{CS} 40 \times \mathrm{CS} 08$ & 0.07 & 0.64 & -0.04 & -0.003 & 0.50 & 0.13 \\
\hline $\mathrm{CS} 40 \times \mathrm{CS} 39$ & $-2.31 * *$ & $-2.24 * *$ & -0.38 & -0.64 & $-2.21 * *$ & $-1.75^{* *}$ \\
\hline $\mathrm{CS} 40 \times \mathrm{CS} 44$ & $2.24 * *$ & $1.61 *$ & $0.41^{*}$ & 0.65 & $1.71^{*}$ & $1.63 *$ \\
\hline $\mathrm{CS} 50 \times \mathrm{CS} 08$ & -0.60 & -0.05 & -0.19 & $3.60 * *$ & -0.61 & 0.79 \\
\hline CS50×CS39 & $2.22 * *$ & 1.07 & 0.20 & -1.44 & $2.35 * *$ & 1.58 \\
\hline $\mathrm{CS} 50 \times \mathrm{CS} 44$ & $-1.62 * *$ & -1.02 & -0.01 & $-2.15^{* *}$ & $-1.74 *$ & $-2.38 * *$ \\
\hline CS51×CS08 & -0.93 & -0.38 & 0.01 & -0.11 & -0.94 & -0.88 \\
\hline CS51×CS39 & $1.35^{*}$ & $1.80 *$ & 0.20 & 0.78 & $2.01 * *$ & $2.25 * *$ \\
\hline CS51×CS44 & -0.42 & -01.42 & -0.21 & -0.66 & -1.07 & $-1.38^{*}$ \\
\hline $\mathrm{CS} 52 \times \mathrm{CS} 08$ & $3.05^{* *}$ & $4.06 * *$ & $0.76^{* *}$ & -0.40 & $3.39 * *$ & $5.46^{* *}$ \\
\hline CS52×CS39 & $-4.80 * *$ & $-6.89 * *$ & $-0.51^{*}$ & -0.04 & $-5.99 * *$ & $-8.42 * *$ \\
\hline CS52×CS44 & $1.76^{* *}$ & $2.83 * *$ & -0.25 & 0.45 & $2.60 * *$ & $2.96 * *$ \\
\hline SE(sca) & 0.61 & 0.79 & 0.21 & 0.82 & 0.76 & 0.64 \\
\hline SE (sij- skl) & 0.87 & 1.12 & 0.29 & 0.41 & 1.07 & 0.90 \\
\hline
\end{tabular}


Table 4.2. Estimates of sca effects of crosses for days to 1st fruit harvest, no. of branches per plant, vine length, fruit length and fruit width of cucumber

\begin{tabular}{|c|c|c|c|c|c|}
\hline \multirow[b]{2}{*}{ Crosses } & Days to $1^{\text {st }}$ & \multirow{2}{*}{$\begin{array}{l}\text { No. of branches per } \\
\text { plant }\end{array}$} & \multicolumn{3}{|c|}{ Fruit } \\
\hline & fruit harvest & & Vine length & length & Fruit width \\
\hline $\mathrm{CS} 07 \times \mathrm{CS} 08$ & $-6.94 * *$ & $1.61 * *$ & $23.94 * *$ & $2.08 * *$ & $0.59 * *$ \\
\hline CS07×CS39 & $5.71 * *$ & $-1.50 * *$ & $-48.99 * *$ & $-1.05^{*}$ & $-0.41 * *$ \\
\hline CS07×CS44 & $1.23^{*}$ & 0.11 & $25.05 * *$ & $-1.02 *$ & -0.18 \\
\hline CS16×CS08 & 0.08 & $-1.21 * *$ & $25.74 * *$ & $-2.71 * *$ & 0.06 \\
\hline CS16×CS39 & $2.97 * *$ & -0.02 & $-16.66^{*}$ & 0.14 & $-0.28 * *$ \\
\hline CS16×CS44 & $-3.05^{* *}$ & $1.24 * *$ & -9.08 & $2.57 * *$ & $0.22 *$ \\
\hline CS17×CS08 & $1.17 *$ & $2.06 * *$ & $24.61 * *$ & $-2.16^{* *}$ & -0.14 \\
\hline CS17×CS39 & $-5.34 * *$ & $1.24 * *$ & $18.21 *$ & $3.09 * *$ & $0.27 * *$ \\
\hline CS17×CS44 & $4.17 * *$ & $-3.30 * *$ & $-42.82 * *$ & $-0.93 *$ & -0.12 \\
\hline CS18×CS08 & 0.44 & 0.17 & $22.27 * *$ & $1.86^{* *}$ & 0.16 \\
\hline CS18×CS39 & $1.92 * *$ & $-1.18 * *$ & $-51.92 * *$ & -0.53 & 0.08 \\
\hline CS18×CS44 & $-2.36 * *$ & $1.01 * *$ & $29.65 * *$ & $-1.33 * *$ & $-0.24^{*}$ \\
\hline $\mathrm{CS} 40 \times \mathrm{CS} 08$ & 0.26 & $-0.54 *$ & -12.71 & $1.94 * *$ & $0.77 * *$ \\
\hline CS40×CS39 & $-2.02 * *$ & $1.11 * *$ & $30.14 * *$ & -0.55 & -0.15 \\
\hline $\mathrm{CS} 40 \times \mathrm{CS} 44$ & $1.76^{* *}$ & $-0.57 *$ & $-17.42 *$ & $-1.39 * *$ & $-0.62 * *$ \\
\hline $\mathrm{CS} 50 \times \mathrm{CS} 08$ & 0.28 & $0.57 *$ & $-23.54 * *$ & -0.04 & $-0.27 * *$ \\
\hline CS50×CS39 & 0.90 & -0.45 & $18.91 *$ & $-1.42 * *$ & $-0.48 * *$ \\
\hline CS50×CS44 & $-1.18^{*}$ & -0.12 & 4.62 & $1.46^{* *}$ & $0.75^{* *}$ \\
\hline CS51×CS08 & 0.93 & 0.41 & $24.70 * *$ & 0.27 & $-0.42 * *$ \\
\hline CS51×CS39 & $2.61 * *$ & $-1.67 * *$ & $-15.97 *$ & $-2.38 * *$ & -0.13 \\
\hline CS51×CS44 & $-3.54 * *$ & $1.26^{* *}$ & -8.73 & $2.11 * *$ & $0.55^{* *}$ \\
\hline CS52×CS08 & $3.77 * *$ & $-3.06 * *$ & $-84.99 * *$ & $-1.22 * *$ & $-0.76^{* *}$ \\
\hline CS52×CS39 & $-6.74 * *$ & $2.46^{* *}$ & $66.28 * *$ & $2.70 * *$ & $1.11 * *$ \\
\hline $\mathrm{CS} 52 \times \mathrm{CS} 44$ & $2.97 * *$ & $0.59 *$ & $18.72 *$ & $-1.48 * *$ & $-0.35 * *$ \\
\hline SE(sca) & 0.58 & 0.29 & 7.43 & 0.42 & 0.10 \\
\hline SE (sij- skl) & 0.82 & 0.41 & 10.50 & 0.59 & 0.14 \\
\hline
\end{tabular}

$*$ and $* *$ significant at $5 \%$ and $1 \%$ level, respectively 
Table 4.3. sca effects of crosses for fruit flesh thickness, single fruit weight, number of fruits per plant, yield per plant and thousand seed weight of cucumber

\begin{tabular}{|c|c|c|c|c|c|}
\hline Crosses & $\begin{array}{c}\text { Flesh } \\
\text { thickness }\end{array}$ & $\begin{array}{l}\text { Single fruit } \\
\text { weight }\end{array}$ & $\begin{array}{c}\text { No. of fruits per } \\
\text { plant }\end{array}$ & $\begin{array}{c}\text { Yield per } \\
\text { plant }\end{array}$ & $\begin{array}{c}\text { Thousand seed } \\
\text { weight }\end{array}$ \\
\hline CS07×CS08 & $0.49 * *$ & $35.77 * *$ & $1.42 * *$ & $0.60 * *$ & $-2.03^{*}$ \\
\hline CS07×CS39 & $-0.16^{* *}$ & $-44.89 * *$ & -0.35 & -0.08 & -1.51 \\
\hline CS07×CS44 & $-0.34 * *$ & 9.12 & $-1.07 * *$ & $-0.52 * *$ & $3.54 * *$ \\
\hline $\mathrm{CS} 16 \times \mathrm{CS} 08$ & $-0.22 * *$ & $30.52 * *$ & $-1.01 * *$ & $-0.53 * *$ & -0.38 \\
\hline CS16×CS39 & $-0.15^{* *}$ & -7.13 & -0.39 & -0.12 & 0.57 \\
\hline CS16×CS44 & $0.38 * *$ & $-23.39 * *$ & $1.40 * *$ & $0.65 * *$ & -0.19 \\
\hline $\mathrm{CS} 17 \times \mathrm{CS} 08$ & $-0.17 * *$ & 7.35 & 0.05 & -0.03 & $3.96 * *$ \\
\hline CS $17 \times$ CS39 & $0.18 * *$ & $16.62 * *$ & $0.78 * *$ & $0.28 *$ & $-3.34 * *$ \\
\hline $\mathrm{CS} 17 \times \mathrm{CS} 44$ & -0.01 & $-23.97 * *$ & $-0.83 * *$ & $-0.25 *$ & -0.61 \\
\hline $\mathrm{CS} 18 \times \mathrm{CS} 08$ & 0.02 & $23.26 * *$ & $0.65^{* *}$ & -0.10 & 0.50 \\
\hline CS $18 \times$ CS39 & 0.09 & $-18.73 * *$ & -0.02 & 0.12 & 1.71 \\
\hline CS $18 \times$ CS44 & $-0.11 *$ & -4.52 & $-0.63 * *$ & -0.02 & -2.21 \\
\hline $\mathrm{CS} 40 \times \mathrm{CS} 08$ & $0.35 * *$ & $30.24 * *$ & $0.79 * *$ & $0.50 * *$ & -0.40 \\
\hline $\mathrm{CS} 40 \times \mathrm{CS} 39$ & $-0.27 * *$ & $-27.36 * *$ & 0.11 & -0.08 & 1.76 \\
\hline $\mathrm{CS} 40 \times \mathrm{CS} 44$ & -0.08 & -2.88 & $-0.90 * *$ & $-0.42 * *$ & -1.36 \\
\hline CS50×CS08 & -0.01 & $-35.45 * *$ & -0.38 & -0.06 & $-3.05 * *$ \\
\hline CS50×CS39 & $-0.23 * *$ & 2.76 & 0.25 & -0.05 & 1.56 \\
\hline CS50×CS44 & $0.24 * *$ & $32.70 * *$ & 0.13 & 0.11 & 1.48 \\
\hline CS51×CS08 & $-0.17 * *$ & $-36.41 * *$ & $-0.91 * *$ & -0.16 & 1.26 \\
\hline CS51×CS39 & -0.04 & $-18.07 * *$ & $-0.59 *$ & $-0.25^{*}$ & $-3.38 * *$ \\
\hline CS51×CS44 & $0.21 * *$ & $54.47 * *$ & $1.50 * *$ & $0.41^{* *}$ & $2.12 *$ \\
\hline CS52 $\times$ CS08 & $-0.29 * *$ & $-55.27 * *$ & $-0.61 * *$ & -0.23 & 0.14 \\
\hline CS $52 \times$ CS 39 & $0.57 * *$ & $96.80 * *$ & 0.21 & 0.18 & $2.63 * *$ \\
\hline $\mathrm{CS} 52 \times \mathrm{CS} 44$ & $-0.29 * *$ & $-41.52 * *$ & 0.40 & 0.05 & $-2.78 * *$ \\
\hline $\mathrm{SE}$ (sca) & 0.06 & 5.06 & 0.23 & 0.13 & 0.92 \\
\hline SE (sij- skl) & 0.08 & 7.16 & 0.33 & 0.19 & 1.30 \\
\hline
\end{tabular}

The sca effects of certain crosses were related with $g c a$ of their parents, as the best cross combinations for most of the characters involved both or at least one parent with high or average gca effects for particular trait. Considering the sca effects the combinations CS07 $\times$ CS08 was best for days to $1^{\text {st }}$ male and female flower, node at $1^{\text {st }}$ male and female flower appearance, days to $50 \%$ plants male and female flower, vine length, number of branches and fruits per plant, days to $1^{\text {st }}$ fruit harvest, fruit length, fruit width, flesh thickness, single fruit weight and yield per plant, CS52 $\times$ CS39 was best for days to $1^{\text {st }}$ male and female flowers, node at $1^{\text {st }}$ male flowers appearance, days to $50 \%$ plants male and female flowers, vine length, number of branches per plant, days to $1^{\text {st }}$ fruit harvest, fruit length, fruit width, flesh thickness, single fruit weight and thousands seed weight, on the other hand, CS16 $\times$ CS44 was best for days to $1^{\text {st }}$ male flowers, node at $1^{\text {st }}$ male flowers appearance, days to $50 \%$ male and female flowers, number of branches and fruits per plant, days to $1^{\text {st }}$ fruit harvest, fruit length, flesh thickness and yield per plant, CS51 $\times$ CS44 was also best for number of branches and fruits per plant, days to $1^{\text {st }}$ fruit harvest, fruit length, fruit width, single fruit weight, thousands seed weight and yield per plant, CS40 $\times$ CS08 was best for number of branches, fruits per plant, fruit length, fruit width, flesh thickness, single fruit weight and yield per plant, CS17 $\times$ CS39 
was best for days to first male and female flowers, days to $50 \%$ male and female flowers, vine length, fruit length and yield per plant found high sca effects which could be exploited for hybrid vigor.

The present findings were in agreement with those reported by Verma et al. (2000) and Singh et al. (1998) in cucumber. Uddin et al. (2009) reported that their crosses showed desirable significant effects in various traits involving high $\times$ high, high $\times$ low, and low $\times$ low combiners indicating the role of additive, dominance and epistatic gene action. Heterosis (high $s c a$ ) in the crosses involving high $\times$ high general combiners might be due to additive $\times$ additive type interaction which was partially fixable. High sca effects in the crosses involving low $\times$ low combining parents were possibly due to intra and inter allelic interaction.

\section{Genetic Component Analysis}

The mean sum of squares due to $g c a$ and $s c a$ were used to estimate the variances for $g c a$ and sca respectively, based on these variances the additive and dominance variances and their ratio are also calculated.

The magnitude of $\sigma^{2} \mathrm{SCA}$ was high in all characters compared to $\sigma^{2} \mathrm{GCA}$ and dominance variance $\left(\sigma^{2} \mathrm{D}\right)$ was higher than the additive genetic variance $\left(\sigma^{2} \mathrm{~A}\right)$ indicating that the predominance of non additive gene action (Table 5.1). Similar result also revealed by Sharma (2010). The estimates of GCA and SCA variances, additive $\left(\sigma^{2} \mathrm{~A}\right)$ and non-additive $\left(\sigma^{2} \mathrm{D}\right)$ components of variance for yield per plant and majority of the important traits, shows that non-additive gene action was in preponderance, which proved the possibility of exploitation of hybrid vigor in cucumber. Uddin et al. (2009) observed the magnitude of $\sigma^{2} \mathrm{SCA}$ high in all characters compared to $\sigma^{2} \mathrm{GCA}$, and dominance variance $\left(\sigma^{2} \mathrm{D}\right)$ was higher than the additive genetic variance $\left(\sigma^{2} \mathrm{~A}\right)$. The ratios of SCA to GCA variance were higher than unity indicating predominated non-additive gene action over additive gene action for all characters. However the prevalence of non-additive gene action and the existence of sizeable amount of additive gene action in the inheritance of yield contributing attributes suggest that transgressive breeding may not be useful for the used parents. Non-additive gene action is prerequisite for launching a heterosis breeding program. Thus heterosis breeding could be the better choice for improvement of cucumber. Prasad et al. (2004) and Uddin et al. (2009) stated similar results in cucumber. 
Table 5.1. Estimation of genetic components of variation and ratios for different characters

\begin{tabular}{|c|c|c|c|c|c|c|c|}
\hline $\begin{array}{l}\text { Sl. } \\
\text { No. }\end{array}$ & Characters & $\sigma^{2} g c a$ & $\sigma^{2} s c a$ & $\sigma^{2}$ additive & $\sigma^{2}$ dominance & $\begin{array}{l}\sigma^{2} \mathrm{D} \\
/ \sigma^{2} \mathrm{~A} \\
\text { ratio } \\
\end{array}$ & $\begin{array}{l}\sigma^{2} s c a / \sigma^{2} \\
\text { gca ratio }\end{array}$ \\
\hline 01. & $\begin{array}{l}\text { Days to } 1^{\text {st }} \text { male } \\
\text { flower }\end{array}$ & 0.60 & 30.20 & 2.40 & 15.52 & 6.47 & 50.33 \\
\hline 02. & $\begin{array}{l}\text { Node at } 1^{\text {st }} \text { first } \\
\text { male flower } \\
\text { appearance }\end{array}$ & 0.003 & 0.51 & 0.01 & 0.96 & 96 & 170 \\
\hline 03. & $\begin{array}{l}\text { Days to } 1^{\text {st }} \\
\text { female flower }\end{array}$ & 0.72 & 44.89 & 2.87 & 25.54 & 8.89 & 62.35 \\
\hline 04. & $\begin{array}{l}\text { Node at } 1^{\text {st }} \\
\text { female flower } \\
\text { appearance }\end{array}$ & 0.002 & 2.49 & 0.01 & 4.41 & 441 & 1245 \\
\hline 05. & $\begin{array}{l}\begin{array}{l}\text { Days to } 50 \% \\
\text { plants } \\
\text { flower }\end{array} \\
\text { male }\end{array}$ & 0.67 & 34.62 & 2.67 & 20.30 & 7.60 & 51.67 \\
\hline 06. & $\begin{array}{l}\text { Days to } \begin{array}{r}50 \% \\
\text { plants } \\
\text { flower }\end{array} \\
\text { female }\end{array}$ & 0.79 & 54.03 & 3.15 & 35.62 & 11.30 & 68.39 \\
\hline 07. & Vine length $(\mathrm{cm})$ & 152.54 & 10125.05 & 610.17 & 3701.00 & 6.06 & 66.37 \\
\hline 08. & $\begin{array}{l}\text { Number of } \\
\text { branches per vine }\end{array}$ & 0.10 & 10.10 & 0.41 & 6.55 & 15.97 & 101 \\
\hline 09. & $\begin{array}{l}\text { Number of fruits } \\
\text { per vine }\end{array}$ & 0.08 & 7.01 & 0.30 & 4.27 & 14.23 & 87.63 \\
\hline 10. & $\begin{array}{l}\text { Days to } 1^{\text {st }} \text { fruit } \\
\text { harvest }\end{array}$ & 0.76 & 50.04 & 3.05 & 30.76 & 10.08 & 65.84 \\
\hline 11. & Fruit length $(\mathrm{cm})$ & 0.18 & 17.62 & 0.72 & 9.99 & 13.87 & 97.88 \\
\hline 12. & Fruit width $(\mathrm{cm})$ & 0.003 & 0.73 & 0.01 & 0.72 & 72 & 243.33 \\
\hline 13. & $\begin{array}{l}\text { Flesh thickness } \\
(\mathrm{cm})\end{array}$ & 0.001 & 0.13 & 0.003 & 0.21 & 70 & 130 \\
\hline 14. & $\begin{array}{ll}\text { Single } & \text { fruit } \\
\text { weight }(\mathrm{g}) & \end{array}$ & 57.82 & 5617.45 & 231.30 & 4162.74 & 17.99 & 97.15 \\
\hline 15. & $\begin{array}{l}\text { Yield per plant } \\
(\mathrm{kg})\end{array}$ & 0.01 & 1.06 & 0.03 & 0.88 & 29.33 & 106 \\
\hline 16. & $\begin{array}{l}1000 \text { seed weight } \\
(\mathrm{g})\end{array}$ & 0.20 & 13.31 & 0.82 & 13.44 & 16.39 & 66.55 \\
\hline
\end{tabular}

\section{Conclusion}

Considering the gca effects, the tasters CS08 and CS44 were best for earliness and other horticultural traits. However, the parental lines CS16 and CS40 were best for earliness. The inbred lines CS51 and CS07 were best for fruit length, fruit breadth, flesh thickness, single fruit weight, fruits per plant and yield per plant. The hybrids CS07×CS08, CS16 $\times$ CS44, CS51 $\times$ CS44, CS40 $\times$ CS08, CS17 $\times$ CS39 were superior in terms of yield per plant and its component characters. The magnitude of $\sigma^{2} \mathrm{SCA}$ was high in all characters compared to $\sigma^{2} \mathrm{GCA}$ and dominance variance $\left(\sigma^{2} \mathrm{D}\right)$ was higher than the additive genetic variance $\left(\sigma^{2} \mathrm{~A}\right)$ indicating that the predominance role of non additive gene action. The results indicated the importance of heterosis breeding for effective utilization of non-additive genetic variance in cucumber. 


\section{References}

Ananthan, M. and C. M. Pappiah. 1997. Combining ability and correlation studies in cucumber (Cucumis sativus L.). South Indian Hort. 45: 1-2, 57-58.

Anonymous. 2012. Nutritional recommendations for cucumber (Cucumis sativus L.) in open fields, tunnel and greenhouse. Haifa, Pioneering the Future. pp 45.

Eifediyi, E. K. and S. U. Remison. 2010. Growth and yield of cucumber (Cucumis sativus L.) as influenced by farmland manure and inorganic fertilizer. Journal of Plant Breeding and Crop Science. 2(7): 216-220.

Fertilizer Recommendation Guide. 2012. Bangladesh Agricultural Research Council, Farmgate, Dhaka. 1215.

Kempthorne, O. 1957. An introduction to genetic statistics, New York, John Wileys and Sons, Inc., London, pp.1-545.

Krishnaprasad, V. S .R. and D. P. Singh. 1992. Combining ability through line $\times$ tester analysis in cucumber (Cucumis sativus L.). Indian J. of Hort. 49(4): 358-362.

Prasad, V.S.R.K., M. Rai, R.S. Pan and A.K. Singh. 2004. Combining ability and standardized potency in crosses between Cucumis sativus L. $\times$ C. hardwickii R. Ind. J. of Hort. 61(2): 128-131.

Ram, D., G. Kalloo and M. Singh. 1999. Combining ability of quantitative characters in bitter gourd ( Momordica charantica). Indian J. Agric. Sci. 69(2): 122-125.

Renner, S. S., H. Schaefer and A. Kocyan. 2007. Phylogenetics of Cucumis (Cucurbitaceae): cucumber (C. sativus) belongs in an Asian/Australian clade far from melon (C. melo). BMC Evolutionary Biology. 7: 58.

Sharma, A., K. Vidyasagar and N. K. Pathania. 2001. Studies on combining ability for earliness marketable fruit yield in cucumber (Cucumis sativus L.). Himachal J. Agric. Res. 58(7): 578-586.

Sharma, M. 2010. Gene action and heterosis studies involving gynoecious lines in cucumber (Cucumis sativus L.) M.S. Thesis, Department of Vegetable Science and Floriculture, Himachal Pradesh Krishi Vishuavidyalaya, Palampur, India.

Singh, P. K., J. C. Kumar and J. R. Sharma. 1998. Heterosis studied in long fruited bottle gourd. Vegetable Sci, 25(1): 55-57.

Tasdighi, M. and L. R. Baker. 1981. Comparison of single and three way crosses of pickling cucumber hybrids for femaleness and yield once over harvest. J. Amer. Soc. Hort. Sci. 106 (3): 370-373.

Uddin, M. N., M. M. Hossain, M. M. Rahman, S. Ahmad and A.K.M. Quamruzzaman. 2009. Combining Ability and Gene Action In Cucumber (Cucumis Sativus L.). SAARC J. Agri. 7 (1), 64-72. 


\section{Macrothink}

Journal of Agricultural Studies

ISSN 2166-0379 2018, Vol. 6, No. 2

Uzodike, E. B. and I. N. Onuoha. 2009. The effect of cucumber (Cucumis Sativus L.) extract on acid induced corneal burn in Guinea Pigs. African Journal Online. 15: 3-5.

Verma, T. S., R. V. Singh and S. C. Sharma. 2000. Line $\times$ tester analysis for combining ability in cucumber. Indian J. of Hort. 2: 144-147.

\section{Copyright Disclaimer}

Copyright for this article is retained by the author(s), with first publication rights granted to the journal.

This is an open-access article distributed under the terms and conditions of the Creative Commons Attribution license (http://creativecommons.org/licenses/by/4.0/). 